\title{
Facile iron(III) chloride hexahydrate catalyzed synthesis of coumarins
}

\author{
Saisuree Prateeptongkum, ${ }^{\mathrm{a}}$ Nongnaphat Duangdee, ${ }^{\mathrm{b}}$ and Panumart Thongyoo ${ }^{\mathrm{a}}$ \\ ${ }^{a}$ Department of Chemistry, Faculty of Science \& Technology, Thammasat University, 99 Moo 18 \\ Paholyothin Road, Klong Luang, Rangsit, Prathumthani 12121, Thailand \\ ${ }^{b}$ Drug Discovery and Development Center, Thammasat University, 99 Moo 18 Paholyothin \\ Road, Klong Luang, Rangsit, Prathumthani 12121, Thailand \\ E-mail:saisuree@tu.ac.th
}

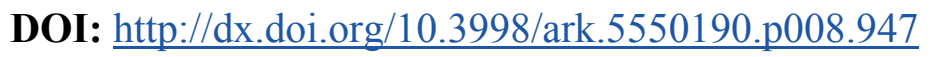

\begin{abstract}
A practical and inexpensive synthesis of coumarins, from phenols and $\beta$-keto esters, via the Pechmann reaction catalyzed by $10 \mathrm{~mol} \%$ of $\mathrm{FeCl}_{3} \cdot 6 \mathrm{H}_{2} \mathrm{O}$ is described. The reaction was applied to transform phenols and $\beta$-keto esters into the corresponding coumarins in moderate to excellent yields.
\end{abstract}

Keywords: Pechmann reaction, coumarin, hydrated Fe(III) chloride, phenols, $\beta$-keto esters

\section{Introduction}

Coumarins are one of the important structural units and widely found in nature. ${ }^{1-4}$ They show diverse biological and pharmacological activities ranging from antimicrobial, anti-arrhythmic, antitumor, antifungal, anti-HIV, anti-osteoporosis to anti-inflammatory. ${ }^{5-12}$ Apart from their pharmaceutical applications, ${ }^{13-14}$ coumarins have been used as additives in foods, perfumes and cosmetics as well as in the preparation of optical brighteners, laser dyes, fluorescent labels and nonlinear optical chromophores. ${ }^{15-19}$

The Pechmann reaction ${ }^{20}$ is known as one of the most valuable methods for the synthesis of coumarins since it proceeds from simple starting materials, a phenol and a $\beta$-keto ester, together with an acid catalyst. Various protocols have been developed by using different catalysts including sulfuric acid, ${ }^{20}$ trifluoroacetic acid, ${ }^{21}$ phosphorus pentaoxide, ${ }^{22}$ aluminium chloride, ${ }^{23-24}$ indium(III) chloride, $^{25}$ titanium(IV) chloride, $^{26}$ silica gel supported zirconyl chloride octahydrate, ${ }^{27}$ samarium(III) nitrate hexahydrate, ${ }^{28}$ bismuth(III) nitrate pentahydrate, ${ }^{29}$ ionic liquids $^{30}$ and many more. ${ }^{31-33}$ However, the development of practical, efficient and environmentally benign synthetic protocols for the synthesis of coumarins is greatly desirable. 
Among the transition metal catalysts, iron is in many ways ideal, owing to its ready availability, low price and environmentally friendly character. In this respect, iron-catalyzed reactions for a variety of transformations have gained an increasing interest in the literature. ${ }^{34-36} \mathrm{~A}$ few examples of iron-catalyzed synthesis of 4-substituted coumarins through the Pechmann condensation have been demonstrated very recently ${ }^{37-38}$ by employing anhydrous $\mathrm{FeCl}_{3}$ as the active catalyst under microwave and ultrasonic irradiation as well as conventional heating ${ }^{37}$ and in an ionic liquid medium. ${ }^{38}$ We envisioned that the use of an Fe(III) hydrate as a catalyst instead of anhydrous $\mathrm{FeCl}_{3}$ would be an advantage, as it is cheap and easily available. There appear to be no reports of the use of such an iron hydrate in the Pechmann reaction. Herein, we report that iron(III) chloride hexahydrate can be used as an effective catalyst for the synthesis of coumarin derivatives by Pechmann condensation of phenols and $\beta$-keto esters.

\section{Results and Discussion}

Initially, the reaction between resorcinol (1a) and methyl acetoacetate (2a) in the presence of iron salt catalyst was selected as a model system.

Table 1. Optimization of the Pechmann reaction conditions ${ }^{\mathrm{a}}$

\begin{tabular}{ccccc} 
& Catalyst $(\mathrm{mol} \%)$ & Solvent & Condition & Yield $(\%)^{\mathrm{b}}$ \\
\hline 1 & $\mathrm{FeCl}_{3} \cdot 6 \mathrm{H}_{2} \mathrm{O}(10)$ & toluene & rt, 16 h & 30 \\
2 & $\mathrm{FeCl}_{3} \cdot 6 \mathrm{H}_{2} \mathrm{O}(10)$ & toluene & reflux, $4.5 \mathrm{~h}$ & 76 \\
3 & $\mathrm{FeCl}_{3} \cdot 6 \mathrm{H}_{2} \mathrm{O}(10)$ & toluene & reflux, 16 h & 92 \\
4 & $\mathrm{FeCl}_{3} \cdot 6 \mathrm{H}_{2} \mathrm{O}(5)$ & toluene & reflux, 16 h & 55 \\
5 & $\mathrm{FeCl}_{3} \cdot 6 \mathrm{H}_{2} \mathrm{O}(20)$ & toluene & reflux, 16 h & 63 \\
6 & $\mathrm{FeCl}_{3} \cdot 6 \mathrm{H}_{2} \mathrm{O}(10)$ & ethanol & reflux, 16 h & 40 \\
7 & $\mathrm{FeSO}_{4} \cdot 7 \mathrm{H}_{2} \mathrm{O}(20)$ & toluene & reflux, 16 h & 20 \\
8 & $\mathrm{NH}_{4} \mathrm{Fe}_{2}\left(\mathrm{SO}_{4}\right)_{2} \cdot 12 \mathrm{H}_{2} \mathrm{O}(10)$ & toluene & reflux, 16 h & 79 \\
\hline
\end{tabular}

a $3 \mathrm{mmol}$ of resorcinol and $3 \mathrm{mmol}$ of methyl acetoacetate in $10 \mathrm{~mL}$ of solvent.

${ }^{\mathrm{b}}$ Isolated yield. 
Various reaction conditions in the presence of different catalytic amounts of $\mathrm{FeCl}_{3} \cdot 6 \mathrm{H}_{2} \mathrm{O}$ in toluene at room temperature and under reflux were optimized (Table 1, entries 1-5). It can be clearly seen from Table 1 that a high yield was achieved with the use of $10 \mathrm{~mol} \% \mathrm{FeCl}_{3} \cdot 6 \mathrm{H}_{2} \mathrm{O}$ in toluene under reflux for $16 \mathrm{~h}$ without the need of an inert atmosphere (Table 1, entry 3). However, a low yield was observed using ethanol as a solvent (Table 1, entry 6). A brief survey of other iron salts, such as $\mathrm{FeSO}_{4} \cdot 7 \mathrm{H}_{2} \mathrm{O}$ and $\mathrm{NH}_{4} \mathrm{Fe}\left(\mathrm{SO}_{4}\right)_{2} \cdot 12 \mathrm{H}_{2} \mathrm{O}$ reveal that $\mathrm{FeCl}_{3} \cdot 6 \mathrm{H}_{2} \mathrm{O}$ was the most active catalyst for the model reaction though $\mathrm{NH}_{4} \mathrm{Fe}\left(\mathrm{SO}_{4}\right)_{2} \cdot 12 \mathrm{H}_{2} \mathrm{O}$ showed significant activity (Table 1, entries 7-8).

Table 2. Synthesis of coumarins via the Pechmann reaction catalyzed by $\mathrm{FeCl}_{3} \cdot 6 \mathrm{H}_{2} \mathrm{O}^{\mathrm{a}}$

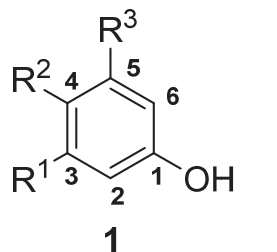

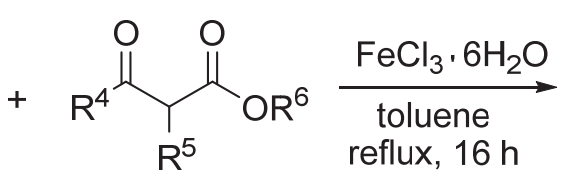

2<smiles>[R]c1cc2oc(=O)c([R8])c([R])c2c([R])c1[R]</smiles>

3

Entry


Table 2. (Continued)

Entry

a $3 \mathrm{mmol}$ of phenol, $3 \mathrm{mmol}$ of methyl acetoacetate and $10 \mathrm{~mol} \%$ of $\mathrm{FeCl}_{3} \cdot 6 \mathrm{H}_{2} \mathrm{O}$ in $10 \mathrm{~mL}$ of toluene, $16 \mathrm{~h}$ under reflux.

${ }^{\mathrm{b}}$ Isolated yield.

Next, the effect of substituents on the reactivity of the phenol were investigated by the reaction of a variety of phenols and methyl acetoacetate (2a) (Table 2, entries 1-6). Phenols with a strong electron-donating group $\left(\mathrm{R}^{1}\right)$ at the meta position yielded the desired products in high yields (Table 2, entries 1-2). These good yields are the result of the presence of the increased electron density at position 6 where electrophilic substitution takes place. 3,5-Disubstituted 
phenols 1c-1e with electron-donating groups gave the corresponding coumarins in lower yields, possibly because of the steric effect of the substituents at position 5 (Table 2, entries 3-5). In addition, 3,4-dimethoxyphenol (1f) showed low activity, leading to $3 \mathrm{f}$ in $44 \%$ yield (Table 2, entry 6). It was assumed that an electron-donating substituent at position 4 could decrease electron density at position 6 .

Finally, the scope and limitations of the reaction were examined by employing various phenols and $\beta$-keto esters (Table 2, entries 7-14). Apart from the substituent effects of the phenols, steric effects from substituents $\left(R^{4}, R^{5}\right)$ of $\beta$-keto esters may play an important role in the reaction as seen more sterically hindered $\beta$-keto esters affording lower yields of the corresponding products (Table 2, entries 7-9, 12-14).

\section{Conclusions}

We have developed a general and practical iron-catalyzed synthetic method for coumarin derivatives via the Pechmann reaction. A variety of phenols and $\beta$-keto esters were converted into the corresponding coumarins in moderate to excellent yields. Notably, the reaction proceeds in the presence of a simple, commercially available and inexpensive iron catalyst.

\section{Experimental Section}

General. Melting points $\left({ }^{\circ} \mathrm{C}\right)$ were measured with a Gallenkamp melting point apparatus and are uncorrected. However, the melting point of $\mathbf{3 1}$ was determined by polarized light microscopy (Olympus BH-2) using a Mettler FP52 microfurnace and FP5 temperature controller. ${ }^{1} \mathrm{H}$ and ${ }^{13} \mathrm{C}$ NMR spectra were recorded on a Bruker AV400 spectrometer. Chemical shifts $(\delta)$ are given in ppm and refer to TMS or the residual undeuterated solvent as the internal standard. The following abbreviations are used: $\mathrm{s}=$ singlet, $\mathrm{d}=$ doublet, $\mathrm{t}=$ triplet, $\mathrm{q}=$ quartet, quint $=$ quintet, sext $=$ sextet, $\mathrm{m}=$ multiplet, $\mathrm{dd}=$ double doublet, br.s $=$ broad singlet. ESI mass spectra were recorded on a Thermo Finnigan LCQ Advantage Mass Spectrometer. High Resolution Mass

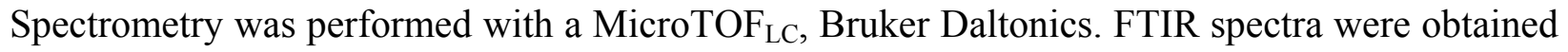
with a Perkin Elmer FT-IR Spectrum GX. Flash chromatography was performed with Fluka silica gel 60 (70-230 mesh) in common glass columns. All chemicals were obtained from commercial suppliers, and were used without further purification.

\section{General procedure for the synthesis of coumarins 3a-3n}

To a mixture of phenol $(3 \mathrm{mmol})$ and $\beta$-keto ester $(3 \mathrm{mmol})$ in toluene $(10 \mathrm{~mL})$ was added iron(III) chloride hexahydrate $(0.08 \mathrm{~g}, 10 \mathrm{~mol} \%)$ at room temperature, and the mixture was heated to reflux for $16 \mathrm{~h}$. After cooling to room temperature, the mixture was quenched with water $(10 \mathrm{~mL})$ and the aqueous phase was extracted with EtOAc $(3 \times 15 \mathrm{~mL})$. The organic phases 
were combined, dried over anhydrous $\mathrm{Na}_{2} \mathrm{SO}_{4}$, concentrated by rotary evaporator and the residue purified by column chromatography on silica gel (eluent: hexane/EtOAc or hexane $/ \mathrm{CH}_{2} \mathrm{Cl}_{2} / \mathrm{Me}_{2} \mathrm{CO}$ ) to give the coumarin product.

7-Hydroxy-4-methyl-2H-2-chromenone (3a). White solid; yield 92\%, mp 188-190 ${ }^{\circ} \mathrm{C}$ (from EtOH-H $\mathrm{H}_{2} \mathrm{O}$ ) (lit ${ }^{37} \mathrm{mp} 184-186{ }^{\circ} \mathrm{C}$ ). IR (nujol): $v_{\max } 3502,2923,2846,1669,1607,1456,1378$, 1277, $1076 \mathrm{~cm}^{-1} .{ }^{1} \mathrm{H}$ NMR $\left(400 \mathrm{MHz}\right.$, DMSO- $\left.d_{6}\right): \delta 10.50(\mathrm{~s}, 1 \mathrm{H}), 7.58(\mathrm{~d}, J 8.7 \mathrm{~Hz}, 1 \mathrm{H}), 6.80$ $(\mathrm{dd}, J 8.7,2.3 \mathrm{~Hz}, 1 \mathrm{H}), 6.70(\mathrm{~d}, J 2.3 \mathrm{~Hz}, 1 \mathrm{H}), 6.11(\mathrm{~d}, J 1.0 \mathrm{~Hz}, 1 \mathrm{H}), 2.37(\mathrm{~d}, J 1.0 \mathrm{~Hz}, 3 \mathrm{H}) .{ }^{13} \mathrm{C}$ NMR (100 MHz, DMSO- $d_{6}$ ): $\delta 161.63(\mathrm{C}), 160.71(\mathrm{C}), 155.33$ (C), $153.90(\mathrm{C}), 127.00(\mathrm{CH})$, $113.32(\mathrm{CH}), 112.50(\mathrm{C}), 110.72(\mathrm{CH}), 102.67(\mathrm{CH}), 18.51\left(\mathrm{CH}_{3}\right) . \mathrm{MS}\left(\mathrm{ESI}^{+}\right), m / z(\%) 177.7$ $\left(\mathrm{M}+\mathrm{H}^{+}, 100\right)$.

7-Methoxy-4-methyl-2H-chromen-2-one (3b). White solid; yield $83 \%$, mp $157-158{ }^{\circ} \mathrm{C}$ (from EtOH- $\mathrm{H}_{2} \mathrm{O}$ ) (lit ${ }^{39} \mathrm{mp} \mathrm{160-161}{ }^{\circ} \mathrm{C}$ ). IR (nujol): $v_{\max } 2923,2854,1732,1608,1457,1376,1287$, 1267, 1215, 1154, 1072, 1026, $856 \mathrm{~cm}^{-1} .{ }^{1} \mathrm{H}-\mathrm{NMR}\left(400 \mathrm{MHz}, \mathrm{CDCl}_{3}\right): \delta 7.52(\mathrm{~d}, J 8.7 \mathrm{~Hz}, 1 \mathrm{H})$, $6.89(\mathrm{~d}, J 8.7,2.5 \mathrm{~Hz}, 1 \mathrm{H}), 6.84(\mathrm{~d}, J 2.5 \mathrm{~Hz}, 1 \mathrm{H}), 6.16(\mathrm{~d}, J 0.8 \mathrm{~Hz}, 1 \mathrm{H}), 3.90(\mathrm{~s}, 3 \mathrm{H}), 2.42(\mathrm{~d}, J$ $0.8 \mathrm{~Hz}, 3 \mathrm{H}) .{ }^{13} \mathrm{C} \mathrm{NMR}\left(100 \mathrm{MHz}, \mathrm{CDCl}_{3}\right): \delta 162.71(C), 161.17(C), 155.39(C), 152.39(C)$, $125.49(\mathrm{CH}), 113.62(\mathrm{C}), 112.25(\mathrm{CH}), 112.02(\mathrm{CH}), 100.92(\mathrm{CH}), 55.71\left(\mathrm{CH}_{3}\right), 18.59\left(\mathrm{CH}_{3}\right)$; $\mathrm{MS}\left(\mathrm{ESI}^{+}\right), m / z(\%) 191.7\left(\mathrm{M}+\mathrm{H}^{+}, 100\right)$.

5,7-Dimethoxy-4-methyl-2H-chromen-2-one (3c). White solid; yield $76 \%, \mathrm{mp} 170-172{ }^{\circ} \mathrm{C}$ (from $\mathrm{EtOH}-\mathrm{H}_{2} \mathrm{O}$ ) $\left(\mathrm{lit}^{37} \mathrm{mp} 170-172{ }^{\circ} \mathrm{C}\right.$ ). IR (nujol): $v_{\max } 2924,2854,1723,1607,1457,1385$, 1353, 1112, $1081 \mathrm{~cm}^{-1} .{ }^{1} \mathrm{H}$ NMR $\left(400 \mathrm{MHz}, \mathrm{CDCl}_{3}\right): \delta 6.44(\mathrm{~d}, J 2.3 \mathrm{~Hz}, 1 \mathrm{H}), 6.29(\mathrm{~d}, J 2.3 \mathrm{~Hz}$, $1 \mathrm{H}), 5.95(\mathrm{~s}, 1 \mathrm{H}), 3.86(\mathrm{~s}, 3 \mathrm{H}), 3.85(\mathrm{~s}, 3 \mathrm{H}), 2.53(\mathrm{~s}, 3 \mathrm{H}) .{ }^{13} \mathrm{C} \mathrm{NMR}\left(100 \mathrm{MHz}, \mathrm{CDCl}_{3}\right): \delta 162.80$ (C), 161.08 (C), 159.15 (C), 157.02 (C), 154.45 (C), $111.42(\mathrm{CH}), 104.93(\mathrm{C}), 95.48(\mathrm{CH}), 93.42$ $(\mathrm{CH}), 55.73\left(\mathrm{CH}_{3}\right), 55.69\left(\mathrm{CH}_{3}\right), 24.17\left(\mathrm{CH}_{3}\right)$; $\mathrm{MS}\left(\mathrm{ESI}^{+}\right), m / z(\%) 221.9\left(\mathrm{M}+\mathrm{H}^{+}, 100\right)$.

5,7-Dihydroxy-4-methyl-2H-chromen-2-one (3d). White solid; yield 75\%, mp 291-294 ${ }^{\circ} \mathrm{C}$ (from EtOH- $\mathrm{H}_{2} \mathrm{O}$ ) (lit ${ }^{37} \mathrm{mp} \mathrm{293-295}{ }^{\circ} \mathrm{C}$ ); IR (nujol): $v_{\max } 3429,2924,2854,1670,1622,1558$, 1457, 1377, 1301, 1162, $825 \mathrm{~cm}^{-1} .{ }^{1} \mathrm{H}-\mathrm{NMR}\left(400 \mathrm{MHz}, \mathrm{DMSO}-d_{6}\right): \delta 10.52(\mathrm{~s}, 1 \mathrm{H}), 10.29(\mathrm{~s}$, $1 \mathrm{H}), 6.26(\mathrm{~d}, J 2.3 \mathrm{~Hz}, 1 \mathrm{H}), 6.17(\mathrm{~d}, J 2.3 \mathrm{~Hz}, 1 \mathrm{H}), 5.85(\mathrm{~s}, 1 \mathrm{H}), 2.49(\mathrm{~s}, 3 \mathrm{H}) ;{ }^{13} \mathrm{C}$ NMR $(100$ MHz, DMSO- $\left.d_{6}\right): \delta 161.00(C), 160.00(C), 157.87(C), 156.45(C), 154.88(C), 108.79(C H)$,

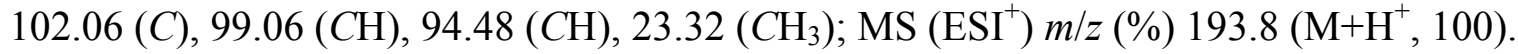

5-Hydroxy-4,7-dimethyl-2H-chromen-2-one (3e). Beige colored solid; yield 46\%, mp 255-257 ${ }^{\circ} \mathrm{C}$ (from EtOH- $\mathrm{H}_{2} \mathrm{O}$ ) $\left(\mathrm{lit}^{28} \mathrm{mp} 257-258{ }^{\circ} \mathrm{C}\right.$ ); IR (nujol): $v_{\max } 3395,2923,1734,1608,1457$, 1377, 1286, $856 \mathrm{~cm}^{-1} .{ }^{1} \mathrm{H}-\mathrm{NMR}\left(400 \mathrm{MHz}, \mathrm{DMSO}-d_{6}\right): \delta 10.52(\mathrm{~s}, 1 \mathrm{H}), 6.63(\mathrm{~s}, 1 \mathrm{H}), 6.58(\mathrm{~s}$, $1 \mathrm{H}), 6.05(\mathrm{~s}, 1 \mathrm{H}), 2.55(\mathrm{~s}, 3 \mathrm{H}), 2.28(\mathrm{~s}, 3 \mathrm{H}) .{ }^{13} \mathrm{C}$ NMR (100 MHz, DMSO- $\left.d_{6}\right): \delta 159.68(C)$, $156.43(\mathrm{C}), 154.78(\mathrm{C}), 154.46(\mathrm{C}), 142.61(\mathrm{C}), 111.89(\mathrm{CH}), 111.76(\mathrm{CH}), 107.58(\mathrm{CH}), 106.47$ (C), $23.30\left(\mathrm{CH}_{3}\right), 21.00\left(\mathrm{CH}_{3}\right)$; $\mathrm{MS}\left(\mathrm{ESI}^{+}\right), m / z(\%) 191.8\left(\mathrm{M}+\mathrm{H}^{+}, 100\right)$.

6,7-Dimethoxy-4-methyl-2H-chromen-2-one (3f). Beige colored solid; yield 44\%, mp 128-129 ${ }^{\circ} \mathrm{C}$ (from EtOH- $\mathrm{H}_{2} \mathrm{O}$ ) $\left(\mathrm{lit}^{40} \mathrm{mp} 130-131{ }^{\circ} \mathrm{C}\right.$, $\mathrm{lit}^{41} \mathrm{mp} \mathrm{136-137}{ }^{\circ} \mathrm{C}$ ). IR (nujol): $v_{\max } 2924,2845$. $1719,1616,1515,1458,1376,1280,1162 \mathrm{~cm}^{-1} .{ }^{1} \mathrm{H}-\mathrm{NMR}\left(400 \mathrm{MHz}, \mathrm{CDCl}_{3}\right): \delta 6.94(\mathrm{~s}, 1 \mathrm{H})$, $6.85(\mathrm{~s}, 1 \mathrm{H}), 6.17(\mathrm{~s}, 1 \mathrm{H}), 3.95(\mathrm{~s}, 3 \mathrm{H}), 3.94(\mathrm{~s}, 3 \mathrm{H}), 2.41(\mathrm{~s}, 3 \mathrm{H}) .{ }^{13} \mathrm{C}$ NMR $\left(100 \mathrm{MHz}, \mathrm{CDCl}_{3}\right)$ : $\delta 161.56(C), 153.03(C), 152.36(C), 149.69$ (C), $146.44(C), 112.72(C), 112.58(C H), 105.61$ 
$(\mathrm{CH}), 100.38(\mathrm{CH}), 56.69\left(\mathrm{CH}_{3}\right), 56.50\left(\mathrm{CH}_{3}\right), 18.98\left(\mathrm{CH}_{3}\right) . \mathrm{MS}\left(\mathrm{ESI}^{+}\right), m / z(\%) 221.9\left(\mathrm{M}+\mathrm{H}^{+}\right.$, 100); HRMS (MALDI-TOF): calcd for $\mathrm{C}_{12} \mathrm{H}_{13} \mathrm{O}_{4}[\mathrm{M}+\mathrm{H}]^{+}: 221.0808$; found: 221.0811 .

2,3-Dihydro-7-hydroxycyclopenta[c]chromen-4(1H)-one (3g). Beige colored solid; yield 54\%, $\mathrm{mp} 241-243{ }^{\circ} \mathrm{C}$ (from EtOH- $\mathrm{H}_{2} \mathrm{O}$ ) (lit ${ }^{42} \mathrm{mp} 248-250{ }^{\circ} \mathrm{C}$ ); IR (nujol) $v_{\max } 3193,2923,1675,1621$, $1457,1377,1307,1149,1075,851,721 \mathrm{~cm}^{-1} .{ }^{1} \mathrm{H}-\mathrm{NMR}$ (400 MHz, DMSO- $\left.d_{6}\right): \delta 10.39(\mathrm{~s}, 1 \mathrm{H})$, $7.42(\mathrm{~d}, J 8.5 \mathrm{~Hz}, 1 \mathrm{H}), 6.79(\mathrm{dd}, J 8.5,2.2 \mathrm{~Hz}, 1 \mathrm{H}), 6.75(\mathrm{~d}, J 2.2 \mathrm{~Hz}, 1 \mathrm{H}), 3.02(\mathrm{t}, J 7.5 \mathrm{~Hz}, 2 \mathrm{H})$, $2.72(\mathrm{t}, J 7.5 \mathrm{~Hz}, 2 \mathrm{H}), 2.09$ (quint, $J 7.5 \mathrm{~Hz}, 2 \mathrm{H}) .{ }^{13} \mathrm{C}$ NMR (100 MHz, DMSO- $d_{6}$ ): $\delta 160.40(C)$, 159.22 (C), $156.57(C), 155.27(C), 126.32(C H), 122.29(C), 112.71(C H), 110.61(C), 102.04$ $(\mathrm{CH}), 31.50\left(\mathrm{CH}_{2}\right), 29.84\left(\mathrm{CH}_{2}\right), 21.96\left(\mathrm{CH}_{2}\right)$. MS $\left(\mathrm{ESI}^{+}\right), \mathrm{m} / z(\%) 203.9\left(\mathrm{M}+\mathrm{H}^{+}, 100\right)$; HRMS (MALDI-TOF): calcd for $\mathrm{C}_{12} \mathrm{H}_{11} \mathrm{O}_{3}[\mathrm{M}+\mathrm{H}]^{+}:$203.0703; found: 203.0698 .

7-Hydroxy-4-propyl-2H-chromen-2-one (3h). Beige colored solid; yield 56\%, mp 131-133 ${ }^{\circ} \mathrm{C}$ (from EtOH- $\mathrm{H}_{2} \mathrm{O}$ ) $\left(\mathrm{lit}^{37} \mathrm{mp} 129-131{ }^{\circ} \mathrm{C}\right.$, $\mathrm{lit}^{43} \mathrm{mp} 132-134{ }^{\circ} \mathrm{C}$ ). IR (nujol) $v_{\max } 3193,2926,1696$, $1616,1563,1457,1376,1140,994,850 \mathrm{~cm}^{-1} .{ }^{1} \mathrm{H}-\mathrm{NMR}\left(400 \mathrm{MHz}, \mathrm{DMSO}-d_{6}\right): \delta 10.52(\mathrm{~s}, 1 \mathrm{H})$, $7.65(\mathrm{~d}, J 8.7 \mathrm{~Hz}, 1 \mathrm{H}), 6.80(\mathrm{dd}, J 8.7,2.3 \mathrm{~Hz}, 1 \mathrm{H}), 7.71(\mathrm{~d}, J 2.3 \mathrm{~Hz}, 1 \mathrm{H}), 6.08(\mathrm{~s}, 1 \mathrm{H}), 2.71(\mathrm{t}, J$ $7.5 \mathrm{~Hz}, 2 \mathrm{H}), 1.63$ (sext, $J 7.5 \mathrm{~Hz}, 2 \mathrm{H}), 0.97(\mathrm{t}, J 7.5 \mathrm{~Hz}, 3 \mathrm{H}) ;{ }^{13} \mathrm{C}$ NMR $\left(100 \mathrm{MHz}, \mathrm{DMSO}-d_{6}\right): \delta$ $161.01(C), 160.38$ (C), 156.89 (C), $155.11(C), 126.34(C H), 112.90(C H), 111.21(C), 109.32$ $(\mathrm{CH}), 102.37(\mathrm{CH}), 32.83\left(\mathrm{CH}_{2}\right), 21.34\left(\mathrm{CH}_{2}\right), 13.65\left(\mathrm{CH}_{3}\right) . \mathrm{MS}\left(\mathrm{ESI}^{+}\right), m / z(\%) 205.9\left(\mathrm{M}+\mathrm{H}^{+}\right.$, 100).

4-Ethyl-7-hydroxy-2H-chromen-2-one (3i). White solid; yield $62 \%$, mp $170-173{ }^{\circ} \mathrm{C}$ (from EtOH- $\left.\mathrm{H}_{2} \mathrm{O}\right)\left(\mathrm{lit}^{43} \mathrm{mp} 175-177^{\circ} \mathrm{C}\right.$ ). IR (nujol): $v_{\max } 3230,2923,1678,1623,1600,1456,1376$, 1231, 1159, 1080, $850 \mathrm{~cm}^{-1} .{ }^{1} \mathrm{H}-\mathrm{NMR}$ (400 MHz, DMSO- $\left.d_{6}\right): \delta 10.51(\mathrm{~s}, 1 \mathrm{H}), 7.64(\mathrm{~d}, J 8.7 \mathrm{~Hz}$, $1 \mathrm{H}), 6.81(\mathrm{dd}, J 8.7,2.3 \mathrm{~Hz}, 1 \mathrm{H}), 6.72(\mathrm{~d}, J 2.3 \mathrm{~Hz}, 1 \mathrm{H}), 6.08(\mathrm{~s}, 1 \mathrm{H}), 2.77(\mathrm{q}, J 7.4 \mathrm{~Hz}, 2 \mathrm{H})$, $1.22(\mathrm{t}, J 7.4 \mathrm{~Hz}, 3 \mathrm{H}) .{ }^{13} \mathrm{C}$ NMR (100 MHz, DMSO- $\left.d_{6}\right): \delta 160.94(C), 160.43(C), 158.27(C)$, $154.95(\mathrm{C}), 126.05(\mathrm{CH}), 112.84(\mathrm{CH}), 111.10(\mathrm{C}), 108.33(\mathrm{CH}), 102.31(\mathrm{CH}), 23.94\left(\mathrm{CH}_{2}\right)$, $12.30\left(\mathrm{CH}_{3}\right)$. MS $\left(\mathrm{ESI}^{+}\right), \mathrm{m} / z(\%) 191.8\left(\mathrm{M}+\mathrm{H}^{+}, 100\right)$.

4-Ethyl-7-methoxy-2H-chromen-2-one (3j). Beige colored solid; yield $34 \%$, mp $115-118{ }^{\circ} \mathrm{C}$

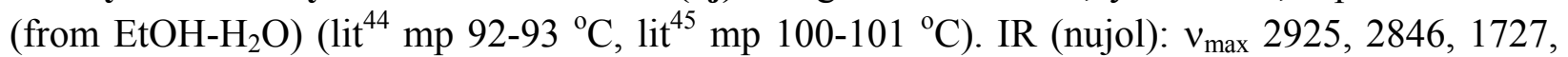
1622, 1457, 1377, 1293, 1153, 1081, 1028, 858, $721 \mathrm{~cm}^{-1} .{ }^{1} \mathrm{H}-\mathrm{NMR}\left(400 \mathrm{MHz}, \mathrm{CDCl}_{3}\right): \delta 7.53$ $(\mathrm{d}, J 8.8 \mathrm{~Hz}, 1 \mathrm{H}), 6.86(\mathrm{dd}, J 8.8,2.4 \mathrm{~Hz}, 1 \mathrm{H}), 6.83(\mathrm{~d}, J 2.4 \mathrm{~Hz}, 1 \mathrm{H}), 6.15(\mathrm{~s}, 1 \mathrm{H}), 3.87(\mathrm{~s}, 3 \mathrm{H})$, 2.78 (q, J 7.4 Hz, 2H), 1.32 (t, J 7.4 Hz, 3H). $\left.{ }^{13} \mathrm{C} \mathrm{NMR} \mathrm{(100} \mathrm{MHz,} \mathrm{CDCl}_{3}\right): \delta 162.71(C), 161.73$ $(C), 157.70(C), 155.69(C), 125.23(\mathrm{CH}), 113.08(C), 112.43(\mathrm{CH}), 110.21(\mathrm{CH}), 101.26(\mathrm{CH})$, $55.87\left(\mathrm{CH}_{3}\right), 24.89\left(\mathrm{CH}_{2}\right), 12.39\left(\mathrm{CH}_{3}\right)$. MS $\left(\mathrm{ESI}^{+}\right), \mathrm{m} / z(\%) 205.7\left(\mathrm{M}+\mathrm{H}^{+}, 100\right)$; HRMS (MALDI-TOF): calcd for $\mathrm{C}_{12} \mathrm{H}_{13} \mathrm{O}_{3}[\mathrm{M}+\mathrm{H}]^{+}$: 205.0859; found: 205.0868.

4-Ethyl-5,7-dimethoxy-2H-chromen-2-one (3k). Light yellow solid; yield $20 \%$, mp $146-148{ }^{\circ} \mathrm{C}$ (from $\mathrm{EtOH}-\mathrm{H}_{2} \mathrm{O}$ ) (lit ${ }^{46} \mathrm{mp} 144-145{ }^{\circ} \mathrm{C}$ ); IR (nujol): $v_{\max } 2924,2846,1713,1620,1600,1457$, 1377, 1238, 1160, $1115 \mathrm{~cm}^{-1} .{ }^{1} \mathrm{H}$ NMR $\left(400 \mathrm{MHz}, \mathrm{CDCl}_{3}\right): \delta 6.46(\mathrm{~d}, J 2.4 \mathrm{~Hz}, 1 \mathrm{H}), 6.31(\mathrm{~d}, J$ $2.4 \mathrm{~Hz}, 1 \mathrm{H}), 6.01(\mathrm{~s}, 1 \mathrm{H}), 3.87(\mathrm{~s}, 3 \mathrm{H}), 3.85(\mathrm{~s}, 3 \mathrm{H}), 2.95(\mathrm{q}, J 7.2 \mathrm{~Hz}, 2 \mathrm{H}), 1.22$ (t, $J 7.2 \mathrm{~Hz}$, 3H). ${ }^{13} \mathrm{C}$ NMR (100 MHz, $\left.\mathrm{CDCl}_{3}\right): \delta 162.64(C), 161.42(C), 159.84(C), 158.79(C), 157.23(C)$, $109.72(\mathrm{CH}), 104.36(\mathrm{C}), 95.60(\mathrm{CH}), 93.62(\mathrm{CH}), 55.75\left(\mathrm{CH}_{3}\right), 55.67\left(\mathrm{CH}_{3}\right), 29.36\left(\mathrm{CH}_{2}\right), 13.53$ 
$\left(\mathrm{CH}_{3}\right)$; $\mathrm{MS}\left(\mathrm{ESI}^{+}\right), \mathrm{m} / z(\%) 235.8\left(\mathrm{M}+\mathrm{H}^{+}, 100\right)$; HRMS (MALDI-TOF): calcd for $\mathrm{C}_{13} \mathrm{H}_{15} \mathrm{O}_{4}$ $[\mathrm{M}+\mathrm{H}]^{+}: 235.0965$; found: 235.0975 .

4-Ethyl-5,7-dihydroxy-2H-chromen-2-one (3I). White solid; yield $60 \%, \mathrm{mp} 248{ }^{\circ} \mathrm{C}$ (dec) (from EtOH- $\mathrm{H}_{2} \mathrm{O}$ ) ( $\left(\mathrm{lit}^{47} \mathrm{mp} 241-243{ }^{\circ} \mathrm{C}\right.$ ). IR (nujol): $v_{\max } 3277,2924,2846,1698,1654,1559,1542$, 1457, 1377, 1277, 1157, 1101, 1031, $825 \mathrm{~cm}^{-1} .{ }^{1} \mathrm{H}$ NMR (400 MHz, DMSO- $\left.d_{6}\right): \delta 10.57(\mathrm{~s}, 1 \mathrm{H})$, $10.28(\mathrm{~s}, 1 \mathrm{H}), 6.28(\mathrm{~d}, J 2.3 \mathrm{~Hz}, 1 \mathrm{H}), 6.19(\mathrm{~d}, J 2.3 \mathrm{~Hz}, 1 \mathrm{H}), 5.84(\mathrm{~s}, 1 \mathrm{H}), 2.92(\mathrm{q}, J 7.3 \mathrm{~Hz}, 2 \mathrm{H})$, $1.17(\mathrm{t}, J 7.3 \mathrm{~Hz}, 3 \mathrm{H}) .{ }^{13} \mathrm{C}$ NMR $\left(100 \mathrm{MHz}, \mathrm{DMSO}-d_{6}\right): \delta 160.92(C), 160.33(C), 160.30(C)$, $157.43(\mathrm{C}), 156.74(\mathrm{C}), 107.28(\mathrm{CH}), 101.35(\mathrm{C}), 99.25(\mathrm{CH}), 94.71(\mathrm{CH}), 28.40\left(\mathrm{CH}_{2}\right), 13.89$ $\left(\mathrm{CH}_{3}\right)$; $\mathrm{MS}\left(\mathrm{ESI}^{+}\right), m / z(\%) 207.9\left(\mathrm{M}+\mathrm{H}^{+}, 100\right)$.

5,7-Dihydroxy-4-propyl-2H-chromen-2-one (3m). Light yellow solid; yield 40\%, mp 239-240 ${ }^{\circ} \mathrm{C}$ (from $\mathrm{EtOH}-\mathrm{H}_{2} \mathrm{O}$ ) $\left(\mathrm{lit}^{37} \mathrm{mp} 241-243{ }^{\circ} \mathrm{C}\right.$ ); IR (nujol): $v_{\max } 3216,2925,2846,1664,1616$, $1553,1561,1459,1377,1270,1154,1098,826 \mathrm{~cm}^{-1} .{ }^{1} \mathrm{H}$ NMR (400 MHz, DMSO- $\left.d_{6}\right): \delta 10.57$ (s, 1H), $10.28(\mathrm{~s}, 1 \mathrm{H}), 6.27(\mathrm{~d}, J 2.3 \mathrm{~Hz}, 1 \mathrm{H}), 6.18(\mathrm{~d}, J 2.3 \mathrm{~Hz}, 1 \mathrm{H}), 5.83(\mathrm{~s}, 1 \mathrm{H}), 2.85$ (t, $J 7.4$ $\mathrm{Hz}, 2 \mathrm{H}), 1.59$ (sext, $J 7.4 \mathrm{~Hz}, 2 \mathrm{H}), 0.95(\mathrm{t}, J 7.4 \mathrm{~Hz}, 3 \mathrm{H}) .{ }^{13} \mathrm{C}$ NMR $\left(100 \mathrm{MHz}\right.$, DMSO- $\left.d_{6}\right): \delta$ 160.90 (C), 160.16 (C), 158.49 (C), 157.41 (C), 156.83 (C), 108.28 (CH), 101.37 (C), 99.26 $(\mathrm{CH}), 94.73(\mathrm{CH}), 37.22\left(\mathrm{CH}_{2}\right), 22.52\left(\mathrm{CH}_{2}\right), 13.80\left(\mathrm{CH}_{3}\right)$; $\mathrm{MS}\left(\mathrm{ESI}^{+}\right), m / z(\%) 221.8\left(\mathrm{M}+\mathrm{H}^{+}\right.$, 100).

2,3-Dihydro-7,9-dihydroxycyclopenta[c]chromen-4(1H)-one (3n). Light yellow solid; yield $28 \%, \mathrm{mp} 250{ }^{\circ} \mathrm{C}$ (decomposed) (from $\mathrm{EtOH}-\mathrm{H}_{2} \mathrm{O}$ ) (lit ${ }^{47} \mathrm{mp} 216-218{ }^{\circ} \mathrm{C}$ ). IR (nujol): $v_{\max } 3199$, 2924, 2846, 1681, 1625, 1557, 1463, 1377, 1286, 1153, 1113, 1082, 840, $739 \mathrm{~cm}^{-1} .{ }^{1} \mathrm{H}$ NMR (400 MHz, DMSO- $d_{6}$ ): $\delta 10.34$ (br.s, 1H), 10.17 (br.s, $\left.1 \mathrm{H}\right), 6.24$ (d, $\left.J 2.2 \mathrm{~Hz}, 1 \mathrm{H}\right), 6.19$ (d, $J 2.2$ $\mathrm{Hz}, 1 \mathrm{H}), 3.21(\mathrm{t}, J 7.6 \mathrm{~Hz}, 2 \mathrm{H}), 2.60(\mathrm{t}, J 7.6 \mathrm{~Hz}, 2 \mathrm{H}), 1.99$ (pent, $J 7.6 \mathrm{~Hz}, 2 \mathrm{H}) .{ }^{13} \mathrm{C}$ NMR $(100$ MHz, DMSO- $\left.d_{6}\right): \delta 160.58(C), 159.33(C), 156.50(C), 156.38(C), 156.23(C), 120.04(C)$, $101.30(\mathrm{C}), 98.52(\mathrm{CH}), 94.11(\mathrm{CH}), 35.55\left(\mathrm{CH}_{2}\right), 28.89\left(\mathrm{CH}_{2}\right), 22.23\left(\mathrm{CH}_{2}\right)$. MS $\left(\mathrm{ESI}^{+}\right), m / z(\%)$ $218.4\left(\mathrm{M}^{+}, 100\right)$.

\section{Acknowledgements}

This research was supported by Thammasat University under the TU research Scholar, Contract No. 2/11/2556. We are grateful to Assoc. Prof. Dr. Sukrit Tantrawong for his helpful discussion. We thank Mr. Pawadhol Chingthongcam for his excellent technical support. We also acknowledge Chulabhorn Research Institute for the HRMS analysis.

\section{References}

1. Fukuda, T.; Sudoh, Y.; Tsuchiya,Y.; Okuda, T.; Fujimori, F.; Igarashi, Y. J. Nat. Prod. 2011, $74,1327$.

http://dx.doi.org/10.1021/np200035m 
2. Mahidol, C.; Kaweetripob, W.; Prawat, H.; Ruchirawat, S. J. Nat. Prod. 2002, 65, 757. http://dx.doi.org/10.1021/np010579u

3. Kwak, J. H.; Lee, K. B.; Schmitz, F. J. J. Nat. Prod. 2001, 64, 1081. http://dx.doi.org/10.1021/np010103a

4. Kang, S. Y.; Lee, K. Y.; Sung, S. H.; Park, M. J.; Kim, Y. C. J. Nat. Prod. 2001, 64, 683. http://dx.doi.org/10.1021/np000441w

5. Chen, Y.; Liu, H.-R.; Liu, H.-S.; Cheng, M.; Xia, P.; Qian, K.; Wu, P.-C.; Lai, C.-Y.; Xia, Y.; Yang, Z.-Y.; Morris-Natschke, S. L.; Lee, K.-H. Eur. J. Med. Chem. 2012, 49, 74. http://dx.doi.org/10.1016/j.ejmech.2011.12.025

6. Touisni, N.; Maresca, A.; McDonald, P. C., Lou, Y.; Scozzafava, A.; Dedhar, S.; Winum, J.Y.; Supuran, C. T. J. Med. Chem. 2011, 54, 8271. http://dx.doi.org/10.1021/jm200983e

7. Tseng, C.-H.; Lin, R.-W.; Chen, Y.-L.; Wang, G.-J.; Ho, M.-L.; Tzeng, C.-C. J. Med. Chem. 2011, 54, 3103 . http://dx.doi.org/10.1021/jm1016494

8. Tang, C.-H.; Yang, R.-S.; Chien, M.-Y.; Chen, C.-C.; Fu, W.-M. Eur J Pharacol. 2008, 579, 40.

http://dx.doi.org/10.1016/j.ejphar.2007.10.01

9. Curir, P.; Galeotti, F.; Dolci, M.; Barile, E.; Lanzotti, V. J. Nat. Prod. 2007, 70, 1668. http://dx.doi.org/10.1021/np070295v

10. El-Seedi, H. R. J. Nat. Prod. 2007, 70, 118. http://dx.doi.org/10.1021/np060444u

11. Kontogiorgis, C. A.; Hadjipavlou-Litina, D. J. J. Med. Chem. 2005, 48, 6400. http://dx.doi.org/10.1021/jm0580149

12. Spino, C.; Dodier, M.; Sotheeswaran, S. Bioorg. Med. Chem. Lett. 1998, 8, 3475. http://dx.doi.org/10.1016/S0960-894X(98)00628-3

13. Masche, U. P.; Rentsch, K.; von Felten, M. A.; Meier, P. J.; Fattinger, K. E. Eur. J. Clin. Pharmacol. 1999, 54, 865. http://dx.doi.org/10.1007/s002280050568

14. Parrish, J.; Fitzpatrick, T.; Tannenbaum, L.; Patak, M. New Eng. J. Med. 1974, $291,1207$. http://dx.doi.org/10.1056/NEJM197412052912301

15. Kennedy, R. O.; Thornes, R. D. In Coumarins: Biology, Applications and Mode of Action; John Wiley and Sons: Chichester, 1997.

16. Koefod, R. S.; Mann, K. R. Inorg. Chem. 1989, 28, 2285. http://dx.doi.org/10.1021/ic00311a009

17. Dorlars, A.; Schellhammer, C. W.; Schroeder, J. Angew. Int., Ed. Engl. 1975, 14, 665.

18. Sheng, R.; Wang, P.; Gao, Y.; Wu, Y.; Liu, W.; Ma, J.; Li, H.; Wu, S. Org. Lett. 2008, 10, 5015.

http://dx.doi.org/10.1021/o1802117p

19. Moylan, C. R. J. Phys. Chem. 1994, 98, 13513. 
http://dx.doi.org/10.1021/j100102a014

20. v. Pechmann, H.; Duisberg, C. Ber. Dtsch. Chem. Ges. 1883, 16, 2119.

21. Woods, L.; Sapp, J. J. Org. Chem. 1962, 27, 3703.

http://dx.doi.org/10.1021/jo01057a519

22. Simmonis, H.; Remmert, P. Chem. Ber. 1914, 47, 2229.

http://dx.doi.org/10.1002/cber.191404702131

23. Sethna, S. M.; Shah, N. M.; Shah, R. C. J. Chem. Soc. 1938, 228.

http://dx.doi.org/10.1039/jr9380000228

24. Woodruff, E. H. Organic Syntheses, Coll. Vol. 3, 1955, 581.

25. Bose, D. S.; Rudradas, A. P.; Babu, M. H. Tetrahedron Lett. 2002, 43, 9195. http://dx.doi.org/10.1016/S0040-4039(02)02266-9

26. Valizadeh, H.; Shockravi, A. Tetrahedron Lett. 2005, 46, 3501. http://dx.doi.org/10.1016/j.tetlet.2005.03.124

27. Karami, B.; Kian, M. Catal. Commun. 2011, 14, 62. http://dx.doi.org/10.1016/j.catcom.2011.07.002

28. Bahekar, S. S.; Shinde, D. B. Tetrahedron Lett. 2004, 45, 7999. http://dx.doi.org/10.1016/j.tetlet.2004.09.013

29. Alexander, V. M.; Bhat, R. P.; Samant, S. D. Tetrahedron Lett. 2005, 46, 6957. http://dx.doi.org/10.1016/j.tetlet.2005.07.117

30. Potdar, M. K.; Mohile, S. S.; Salunkhe, M. M. Tetrahedron Lett. 2001, 42, 9285. http://dx.doi.org/10.1016/S0040-4039(01)02041-X

31. Heravi, M. M.; Sadjadi, S.; Oskooie, H. A.; Shoar, R. H.; Bamoharram, F. F. Catal. Commun. 2008, 9, 470.

http://dx.doi.org/10.1016/j.catcom.2007.07.005

32. Chavan, S. P.; Shivasankar, K.; Sivappa, R.; Kale, R. Tetrahedron Lett. 2002, 43, 8583. http://dx.doi.org/10.1016/S0040-4039(02)02006-3

33. Frère, S.; Thiéry, V.; Besson, T. Tetrahedron Lett. 2001, 42, 2791. http://dx.doi.org/10.1016/S0040-4039(01)00295-7

34. Plietker, B. in Iron Catalysis in Organic Chemistry: Reactions and Applications; WileyVCH: Weinheim, 2008. http://dx.doi.org/10.1002/9783527623273

35. Czaplik, W. M.; Mayer, M.; Cvengros, J.; von Wangelin A. J. ChemSusChem 2009, 2, 396. http://dx.doi.org/10.1002/cssc.200900055

36. Bolm, C.; Legros, J.; Paih, J. L.; Zani, L. Chem. Rev. 2004, 104, 6217. http://dx.doi.org/10.1021/cr040664h

37. Prousis, K. C.; Avlonitis, N.; Heropoulos, G. A.; Calogeropoulou, T. Ultrason. Sonochem. 2014, 21, 937. http://dx.doi.org/10.1016/j.ultsonch.2013.10.018

38. Kumar, V.; Tomar, S.; Patel, R.; Yousaf, A.; Parmar, V. S.; Malhotra, S. V. Synth. Commun. 2008, 38, 2646. 


\section{http://dx.doi.org/10.1080/00397910802219569}

39. Kumar, S.; Saini, A.; Sandhu, J. S. Arkivoc 2007, (xv), 18. http://dx.doi.org/10.3998/ark.5550190.0008.f03

40. Al-bayati, R. I. H.; Ayoub, M. T.; Al-hamdany, R. J. Iraqi Chem. Soc. 1985, 10, 141.

41. Ahluwalia, V. K.; Devendra Kumar Indian J. Chem., Sect. B, 1977, 15, 514.

42. Chen, Y.; Lan, Y.; Wang, S.; Zhang, H.; Xu, X.; Liu, X.; Yu, M.; Liu, B.-F.; Zhang, G. Eur. J. Med. Chem. 2014, 74, 427.

http://dx.doi.org/10.1016/j.ejmech.2014.01.012

43. Chen, Y.; Wang, S.; Xu, X.; Liu, X.; Yu, M.; Zhao, S.; Liu, S.; Qiu, Y.; Zhang, T.; Liu, B.F.; Zhang, G. J. Med. Chem. 2013, 56, 4671. http://dx.doi.org/10.1021/jm400408r

44. Ito, K.; Nakajima, K. J. Heterocycl. Chem. 1988, 25, 511. http://dx.doi.org/10.1002/jhet.5570250229

45. Ahluwalia, V. K.; Sunita Indian J. Chem., Sect. B 1977, 15, 240.

46. Hata, K. Yakugaku Zasshi 1956, 76, 666.

47. Ma, T.; Liu, L.; Xue, H.; Li, L.; Han, C.; Wang, L.; Chen, Z.; Liu, G. J. Med. Chem. 2008, $51,1432$.

http://dx.doi.org/10.1021/jm701405p 